\title{
The new exponential identities
}

\section{Mohammed Abdulla Saeed Salem}

\section{Department of Mathematics, College of Education-Radfan, Aden University, Yemen, E. mail: alhoshiby@ hotmail.com}

Recived : $13 \backslash 6 \backslash 2018$

Revised : 2617\2018

Accepted : $118 \backslash 2018$

Available online : $\quad$ 6/8/2018

DOI: 10.29304/jqcm.2018.10.3.410

ABSTRACT: We have obtained new exponential identities. By ten original propositions we have proved them.

Keywords: Identities, Pascal's triangle, Binomial coefficients.

\section{Mathematics subject classification: $11 D 61$.}

\section{Introduction}

Pascal's triangle can be arranged in a triangular array of numbers, as follows:

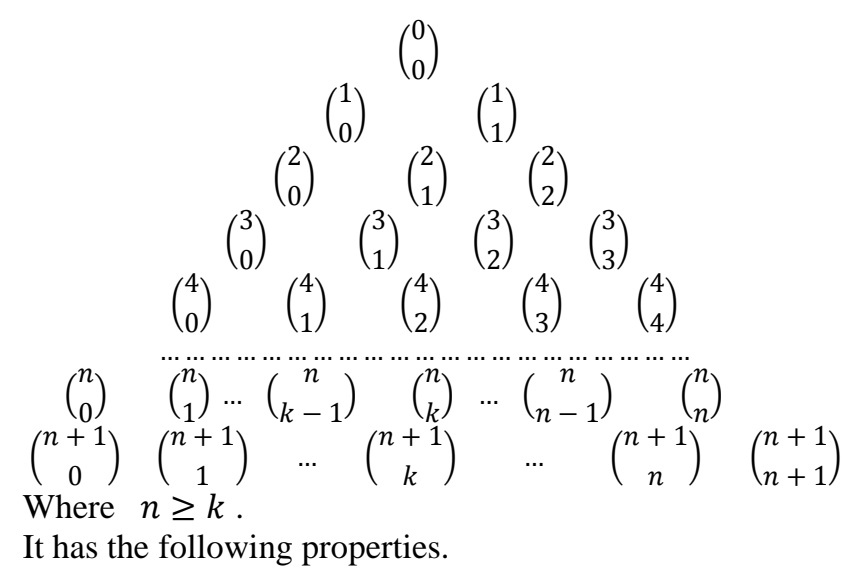

- The first number and the last number in each row is 1.

- Every other number in the array can be obtained by adding the two numbers appearing directly above it. This property is equivalent to the following identity:

$\left(\begin{array}{c}n \\ n-1\end{array}\right)+\left(\begin{array}{l}n \\ k\end{array}\right)=\left(\begin{array}{c}n+1 \\ k\end{array}\right)$

- The numbers equidistant from the ends are equal. This property is equivalent to the following identity:

$\left(\begin{array}{l}n \\ k\end{array}\right)=\left(\begin{array}{c}n \\ n-k\end{array}\right)$ 
Now since the numbers appearing in Pascal's triangle are the binomial coefficients, and here is some of identities satisfied by them.

$$
\begin{aligned}
& \sum_{k=0}^{n}\left(\begin{array}{l}
n \\
k
\end{array}\right)=2^{n} \\
& \sum_{k=0}^{n} k\left(\begin{array}{l}
n \\
k
\end{array}\right)=n 2^{n-1} \\
& \sum_{k=0}^{n}(-1)^{k}\left(\begin{array}{l}
n \\
k
\end{array}\right)=0
\end{aligned}
$$

See [3] for more details.

Can we obtain new identities?

By using the identities above. This paper has answered this question by ten original propositions.

\section{Notation and Definitions}

We denote the set of natural numbers $\mathbb{N}:=$ $\{1,2,3, \ldots\}$. By $\mathbb{Z}$ we denote the set of integers numbers. By $\mathbb{C}$ we denote the complex numbers. The set of $\mathbb{C}^{*}$ is defined by $\mathbb{C}^{*}:=\{z \in \mathbb{C}, z \neq$ $0\}$. The set of all nonzero polynomials over the set $\mathbb{C}$ with indeterminate $z$ is denoted by $\mathbb{C}[z]$. Thus $\mathbb{C}[z]:=\{f(z): f(z)$ is a polynomial,$f(z) \neq 0\}$.

Definition 2.1. (See [1]). A number $P$ is called a composite prime, and $P \in \mathbb{Z}$, if $P=p_{1} \cdot p_{2} \cdots p_{i}$. Where $p_{1}, p_{2}, \ldots, p_{i}$ are distinct primes.

Definition 2.2. (See [2]). We call $\mathbb{A}$ a set of basic numbers.

$$
\begin{aligned}
\mathbb{A}:=\{p, P \in \mathbb{Z}: & p \text { is a prime number, } \\
& P \text { is a composite prime }\} .
\end{aligned}
$$

Definition 2.3. (See [2]). A number $a$ is called a basic number if $a \in \mathbb{A}$.

Definition 2.4 (See [2]). A polynomial $P(z)$ is called a composite primary polynomial, and $P(z) \in \mathbb{C}[z]$, if $P(z)=c p_{1}(z) \cdot p_{2}(z) \cdot p_{i}(z)$. Where $p_{1}(z), p_{2}(z), \ldots, p_{i}(z)$ are irreducible distinct polynomials and $c \neq 0$ is a constant.

Definition 2.5. (See [2]). We call $\mathbb{A}[z]$ a set of basic polynomials.

$$
\begin{aligned}
\mathbb{A}[z]:=\{p(z), P(z) \in \mathbb{C}[z]: p(z) \text { is } a \\
\text { irreducible polynomial, } \\
P(z) \text { is a composite primary } \\
\text { polynomial }\} .
\end{aligned}
$$

Definition 2.6. (See [2]). A polynomial $A(z)$ is called a basic polynomial if $A(z) \in \mathbb{A}[z]$.
Definition 2.7. We will define two types powers triangle:

- A powers triangle of number can be obtained by $z^{r}$ (or $a$ ), where $z \in \mathbb{C}^{*}$ and $r \in \mathbb{N}$, as follows:

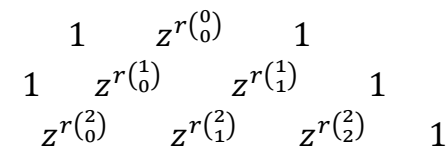

$$
\begin{aligned}
& \begin{array}{lllllll}
1 & z^{r\left(\begin{array}{l}
n \\
0
\end{array}\right)} & \ldots & z^{r\left(\begin{array}{c}
n \\
k-1
\end{array}\right)} & z^{r\left(\begin{array}{c}
n \\
k
\end{array}\right)} & \ldots & z^{r\left(\begin{array}{l}
n \\
n
\end{array}\right)} \quad 1
\end{array}
\end{aligned}
$$

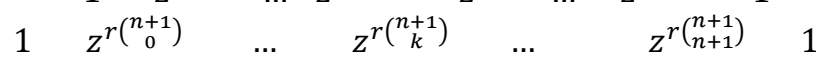

$$
\begin{aligned}
& \mathrm{Or}
\end{aligned}
$$

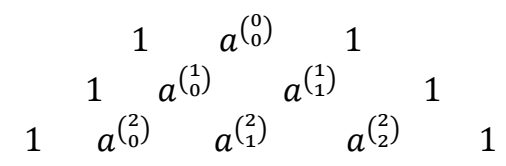

$$
\begin{aligned}
& \begin{array}{lllllll}
1 & a^{\left(\begin{array}{c}
n \\
0
\end{array}\right)} & \ldots & a^{\left(\begin{array}{c}
n \\
k-1
\end{array}\right)} & a^{\left(\begin{array}{c}
n \\
k
\end{array}\right)} & \ldots & a^{\left(\begin{array}{c}
n \\
n
\end{array}\right)} \quad 1
\end{array} \\
& 1 \quad a^{\left(\begin{array}{c}
n+1 \\
0
\end{array}\right)} \quad \ldots \quad a^{\left(\begin{array}{c}
n+1 \\
k
\end{array}\right)} \quad \ldots \quad \ldots \quad a^{\left(\begin{array}{c}
n+1 \\
n+1
\end{array}\right)} \quad 1
\end{aligned}
$$

It has the following interesting properties:

- The first number and the last number in each row is 1 .

- Every other number in the array can be obtained by multiplying the two numbers appearing directly above it.

- The numbers equidistant from the ends are equal.

- A powers triangle of polynomial can be obtained by $f(z)^{r}$ (or $\left.A(z)\right)$. By using the symbol $f(z)$ (or $A(z))$ instead of $z$ (or $a$ ), likewise, we define a powers triangle of polynomial.

\section{The Results}

We have proved the following Results:

\section{Proposition 3.1.}

$$
z^{r\left(\begin{array}{c}
n+1 \\
k
\end{array}\right)}=z^{r\left(\begin{array}{c}
n \\
n-1
\end{array}\right)+r\left(\begin{array}{c}
n \\
k
\end{array}\right) .}
$$

Corollary. If $z^{r}=a$, then

$$
a^{\left(\begin{array}{c}
n+1 \\
k
\end{array}\right)}=a^{\left(\begin{array}{c}
n \\
n-1
\end{array}\right)+\left(\begin{array}{c}
n \\
k
\end{array}\right) .}
$$

Proposition 3.2.

$$
z^{r\left(\begin{array}{l}
n \\
k
\end{array}\right)}=z^{r\left(\begin{array}{c}
n \\
n-k
\end{array}\right) .}
$$

Corollary. If $z^{r}=a$, then

$$
a^{\left(\begin{array}{c}
n \\
k
\end{array}\right)}=a^{\left(\begin{array}{c}
n \\
n-k
\end{array}\right)} .
$$




\section{Proposition 3.3.}

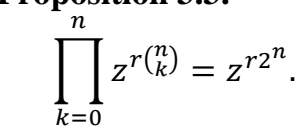

Corollary. If $z^{r}=a$, then

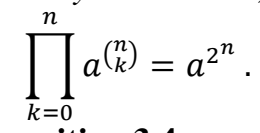

Proposition 3.4.

$$
\prod_{k=0}^{n}\left(z^{\left.r\left(\begin{array}{l}
n \\
k
\end{array}\right)\right)^{k}}=z^{r n 2^{n-1}}\right.
$$

Corollary. If $z^{r}=a$, then

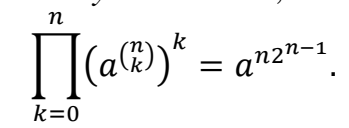

Proposition 3.5.

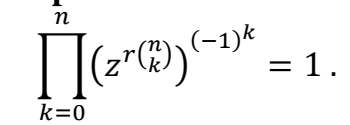

Corollary. If $z^{r}=a$, then

$$
\prod_{k=0}^{n}\left(a^{\left(\begin{array}{c}
n \\
k
\end{array}\right)}\right)^{(-1)^{k}}=1 \text {. }
$$

Proposition 3.6.

$$
f(z)^{r\left(\begin{array}{c}
n+1 \\
k
\end{array}\right)}=f(z)^{r\left(\begin{array}{c}
n \\
n-1
\end{array}\right)+r\left(\begin{array}{c}
n \\
k
\end{array}\right) .}
$$

Corollary. If $f(z)^{r}=A(z)$, then

$$
A(z)^{\left(\begin{array}{c}
n+1 \\
k
\end{array}\right)}=A(z)^{\left(\begin{array}{c}
n \\
n-1
\end{array}\right)+\left(\begin{array}{c}
n \\
k
\end{array}\right) .}
$$

Proposition 3.7.

$$
f(z)^{r\left(\begin{array}{c}
n \\
k
\end{array}\right)}=f(z)^{r\left(\begin{array}{c}
n \\
n-k
\end{array}\right)} .
$$

Corollary. If $f(z)^{r}=A(z)$, then $A(z)^{\left(\begin{array}{c}n \\ k\end{array}\right)}=A(z)^{\left(\begin{array}{c}n \\ n-k\end{array}\right) .}$

Proposition 3.8.

$$
\prod_{k=0}^{n} f(z)^{r\left(\begin{array}{l}
n \\
k
\end{array}\right)}=f(z)^{r 2^{n}}
$$

Corollary. If $f(z)^{r}=A(z)$, then

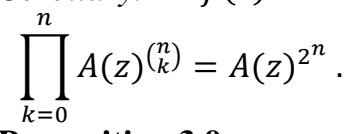

\section{Proposition 3.9.}

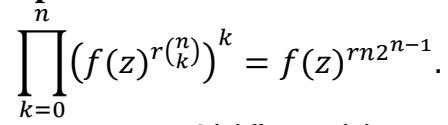

Corollary. If $f(z)^{r}=A(z)$, then

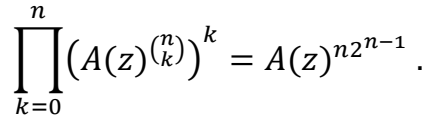

Proposition 3.10.

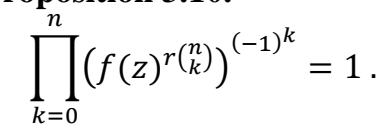

Corollary. If $f(z)^{r}=A(z)$, then

$$
\prod_{k=0}^{n}\left(A(z)^{\left(\begin{array}{l}
n \\
k
\end{array}\right)}\right)^{(-1)^{k}}=1 .
$$

Example 3.1. If a powers triangle is

$$
\begin{array}{cccccccccccc} 
& & & 1 & 4 & 1 & & & \\
& & & & 4 & 4 & 1 & & \\
& & & & 4 & 4^{2} & 4 & 1 & & \\
& & & & 4 & 4^{3} & 4^{3} & 4 & 1 & \\
& & 4 & & 4^{4} & & 4^{6} & 4^{4} & 4 & 1
\end{array}
$$

Compute

a)

$$
\prod_{k=0}^{5} 4\left(\begin{array}{l}
5 \\
k
\end{array}\right)
$$

b)

$$
\prod_{k=0}^{5}\left(4\left(\begin{array}{l}
5 \\
k
\end{array}\right)\right)^{k}
$$

c)

$$
\prod_{k=0}^{5}\left(4\left(\begin{array}{l}
5 \\
k
\end{array}\right)\right)^{(-1)^{k}}
$$

Solution:

a)

b)

$$
\prod_{k=0}^{5} 2^{2\left(\begin{array}{l}
5 \\
k
\end{array}\right)=2^{64} .}
$$

c)

$$
\prod_{k=0}^{5}\left(2^{\left.2\left(\begin{array}{l}
5 \\
k
\end{array}\right)\right)^{k}}=2^{160}\right.
$$

$$
\prod_{k=0}^{5}\left(2^{\left.2\left(\begin{array}{l}
5 \\
k
\end{array}\right)\right)^{(-1)^{k}}}=1\right.
$$

Example 3.2. If a powers triangle is

$$
\begin{aligned}
& \begin{array}{lll}
1 & 3 & 1
\end{array} \\
& \begin{array}{llll}
1 & 3 & 3 & 1
\end{array} \\
& \begin{array}{lllll}
1 & 3 & 3^{2} & 3 & 1
\end{array} \\
& \begin{array}{llllll}
1 & 3 & 3^{3} & 3^{3} & 3 & 1
\end{array} \\
& \begin{array}{lllllll}
1 & 3 & 3^{4} & 3^{6} & 3^{4} & 3 & 1
\end{array}
\end{aligned}
$$

Compute

d)

$$
\prod_{k=0}^{4} 3^{\left(\begin{array}{l}
4 \\
k
\end{array}\right)}
$$

e)

$$
\prod_{k=0}^{4}\left(3\left(\begin{array}{l}
4 \\
k
\end{array}\right)\right)^{k}
$$


Mohammed .A

f)

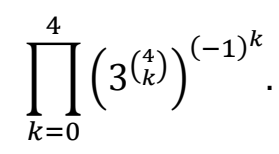

Solution:

d)

$$
\prod_{k=0}^{4} 3^{\left(\begin{array}{l}
4 \\
k
\end{array}\right)=3^{16}}
$$

e)

$$
\left.\prod_{k=0}^{4}\left(3^{4} \begin{array}{l}
4 \\
k
\end{array}\right)\right)^{k}=3^{32}
$$

f)

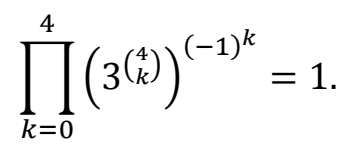

Example 3.3. Compute

a)

$$
\prod_{k=0}^{5}\left(4 z^{2}+2 z+1\right)^{\left(\begin{array}{l}
5 \\
k
\end{array}\right)}
$$

b)

$$
\prod_{k=0}^{5}\left(\left(4 z^{2}+2 z+1\right)^{\left.\left(\begin{array}{l}
5 \\
k
\end{array}\right)\right)^{k}}\right.
$$

c)

$$
\prod_{k=0}^{5}\left(\left(4 z^{2}+2 z+1\right)^{\left.\left(\begin{array}{l}
5 \\
k
\end{array}\right)\right)^{(-1)^{k}} .}\right.
$$

Solution:

a)

$$
\prod_{k=0}^{5}(2 z+1)^{2\left(\begin{array}{l}
5 \\
k
\end{array}\right)}=(2 z+1)^{64}
$$

b)

$$
\prod_{k=0}^{5}\left((2 z+1)^{\left.2\left(\begin{array}{l}
5 \\
k
\end{array}\right)\right)^{k}=(2 z+1)^{160} .}\right.
$$

c)

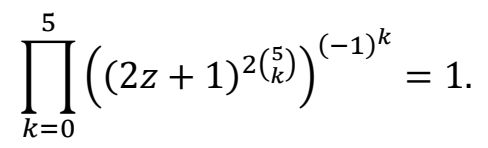

Example 3.4. Compute

a)

$$
\prod_{k=0}^{4}(2 z+1)^{\left(\begin{array}{l}
4 \\
k
\end{array}\right)}
$$

b)

$$
\prod_{k=0}^{4}\left((2 z+1)^{\left.\left(\begin{array}{l}
4 \\
k
\end{array}\right)\right)^{k}}\right.
$$

c)

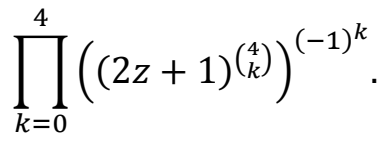

Solution:

a)

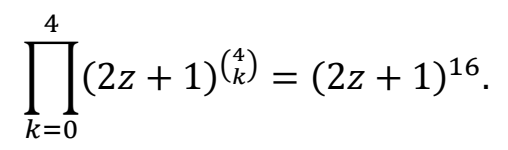

b)

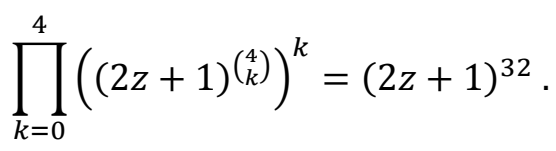

c)

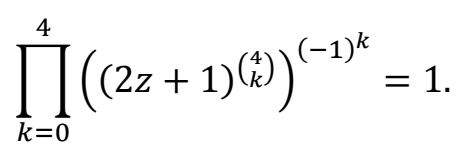

\section{Proof of the Results}

Proof of Proposition 3.1. Since $z=z$, now (1.1) leads to

$$
z^{r\left(\begin{array}{c}
n+1 \\
k
\end{array}\right)}=z^{r\left(\begin{array}{c}
n \\
n-1
\end{array}\right)+r\left(\begin{array}{c}
n \\
k
\end{array}\right)} .
$$

$\square$

Proof of Proposition 3.2. Since $z=z$, now (1.2) leads to

$$
z^{r\left(\begin{array}{c}
n \\
k
\end{array}\right)}=z^{r\left(\begin{array}{c}
n \\
n-k
\end{array}\right)}
$$

Proof of Proposition 3.3. By definition 2.7, in row n

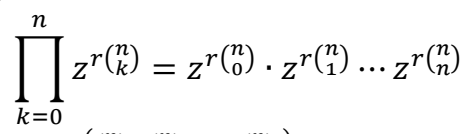

$$
\begin{aligned}
& =z^{r\left(\left(\begin{array}{c}
n \\
0
\end{array}\right)+\left(\begin{array}{l}
n \\
1
\end{array}\right)+\cdots+\left(\begin{array}{l}
n \\
n
\end{array}\right)\right)} \\
& =z^{r 2^{n}} \text {. }
\end{aligned}
$$

Proof of Proposition 3.4. We expand the left-hand side of (3.7)

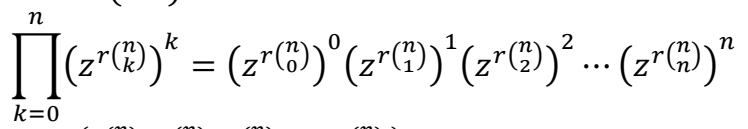

$$
\begin{aligned}
& =z^{r\left(0\left(\begin{array}{l}
n \\
0
\end{array}\right)+1\left(\begin{array}{l}
n \\
1
\end{array}\right)+2\left(\begin{array}{l}
n \\
2
\end{array}\right)+\cdots+n\left(\begin{array}{l}
n \\
n
\end{array}\right)\right)} \quad \text { [By (1.4) ] } \\
& =z^{r n 2^{n-1}} \text {. }
\end{aligned}
$$


Mohammed .A

Proof of Proposition 3.5. We expand the left-hand side of (3.9)

$$
\begin{aligned}
& \prod_{k=0}^{n}\left(z^{r\left(\begin{array}{l}
n \\
k
\end{array}\right)}\right)^{(-1)^{k}}
\end{aligned}
$$

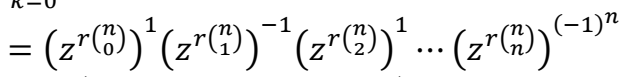

$$
\begin{aligned}
& =z^{r\left(\left(\begin{array}{l}
n \\
0
\end{array}\right)-\left(\begin{array}{c}
n \\
1
\end{array}\right)+\left(\begin{array}{c}
n \\
2
\end{array}\right)+\cdots+(-1)^{n}\left(\begin{array}{l}
n \\
n
\end{array}\right)\right)} \\
& =1 \text {. }
\end{aligned}
$$

Proof. By using the symbol $f(z)$ instead of $z$, likewise, we prove propositions $3.6,3.7,3.8,3.9$ and 3.10 .

\section{Acknowledgements}

To my lovely wife Areefa and my son Qys. Thank you. Without you, I would have never achieved my paper.

\section{References}

[1] Mohammed Abdulla Saeed Salem., "the divisors sequences with finite differences", Journal for Algebra and Number Theory Academia, Mili Publications, 4 (2): pp. 31-39 (2014).

[2] Mohammed Abdulla Saeed Salem., "The special case for divisors sequences with finite differences", Journal for Algebra and Number Theory Academia, Mili Publications, 7 (2): pp.11-36 (2017).

[3] Seymour Lipschutz, Schaum's Outlines of Theory and Problems of Finite Mathematics, first Edition, McGraw-Hill, (1966), pp. 141-144.

\title{
المتطابقات الأسية الجديدة
}

\author{
محمد عبدالله سعيا سالم

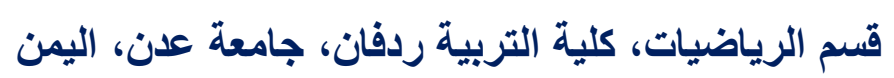

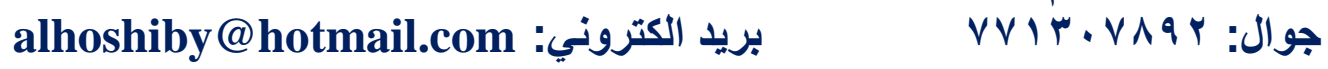

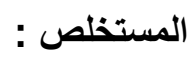 \\ تحصلنا على منطابقات أسية جديدة اثتنتاها بعشر مبر هنات أصلية. \\ كلمات مفتاحية: متطابقات، منلث باسكال، معاملات ذات الحدين.
}

